\title{
Piezoceramic Materials for Ultrasonic Probes
}

\author{
A. M. Abdel Fattah ${ }^{(1)}$, M.G.S.Ali ${ }^{(2)}$, N. Z. ELsyed ${ }^{(2)}$, Gharieb. A. Ali ${ }^{(1)}$ \\ (1) National Institute for Standards NIS-Egypt \\ ${ }^{(2)}$ Menia University - Faculty of Science -Egypt
}

Conventional design for ultrasonic probes tends to favor piezoelectric materials with high electromechanical coupling coefficient. Since the probe performance extremely depends on parameters of the used piezoceramic materials, static and dynamic behaviors of PZT-5H piezoceramic mixture are investigated to assessing its fitness for the criteria of probe designation. The impedance of seven selected PZT-5H piezoceramic plates were analyzed theoretically and experimentally against frequency band of $7 \mathrm{MHz}$ to generate extensive data on characteristics of such ceramics plates. Effect of mechanical and dielectric losses on such impedance was also examined. This may be achieved by introducing the complex constant. Variation of impedances, which represented in circuit model, and the resultant lumped component of equivalent circuit may interpreted the loss mechanisms of such materials that greatly impact a probe's efficiency, maximum drive-level and resonant characteristics.

\section{Introduction:}

The use of piezoceramic materials to produce ultrasonic energy for research and commercial purposes has increased rapidly in recent years. Such materials have the property of reacting mechanically to an applied electrical stimulus, or, reciprocally, of reacting electrically to an applied mechanical stimulus. Such bilateral conversion (transduction) is the function of piezoceramic materials in its various applications. Hence, any analysis of such materials must use electrical and mechanical systems principles.

Piezoceramic materials held a key position as electroacoustic transducers that are used in the construction of ultrasonic probe up to gigahertz. Different piezoceramic compositions of different piezoelectric characteristics were developed. Piezoceramic materials compositions based on lead titanatezirconate $(P Z T)$ offer a wide range of desirable properties than compositions

Momaahlam@yahoo.com 
based on barium titanate, lead metaniobate or sodium niobate [1] This is due to their intrinsically strong piezoelectric effect, high Curie point, and higher coupling coefficient, which allow a wide variation in chemical composition to obtain a wide range of operating parameters without serious reduction of its piezoelectricity. Therefore it is a more desirable choice for the great majority of applications.

Piezoceramic material of particular interest, in this work, is the PZT-5H mixture, since it has been used extensively in ultrasonic probes. These materials offer high thickness coupling coefficient $\mathrm{k}_{\mathrm{t}}$, which correspondence to higher sensitivity .The relatively low clamped dielectric constant $\varepsilon_{\mathrm{S}}$ of PZT-5H makes it suitable for large aperture pulsed devices [2].

\section{Transduction and Piezoelectric Relations:}

Transduction in piezoelectric materials involves the interaction between the electrical and mechanical behavior of such materials. To a good approximation (small signal level) this interaction can be mathematically described by a linear relation between mechanical and electrical variables (constitutive relations). The basic constitutive relations can be expressed in four different forms as follows;

$$
\begin{array}{lll}
D=\varepsilon^{T} E+d T & \text { (1.a) } & D=\varepsilon^{S} E+e S \\
S=s^{E} T+d E & & T=c^{E} S-e E \\
E=\beta^{T} D-g T & & \\
S=s^{D} T+g D & \text { (1.c) } & E=\beta^{S} D-h S \\
& & T=c^{D} S-h D
\end{array}
$$

where, $\beta, \varepsilon$ are dielectric constants, $\mathrm{d}, \mathrm{g}, \mathrm{e}, \mathrm{h}$ are piezoelectric constants, and $\mathrm{s}$, $c$ are elastic constant. The description meaning of these symbols and their units are given in Table (1). The utility of any pairs depends on the fact that certain variables on the right hand are approximately zero under appropriate conditions such as resonator geometry, mechanical and electrical conditions.

In the above equations the electrical quantities $(\mathrm{E}, \mathrm{D})$ have vector nature while the mechanical quantities $(T, S)$ have tensor nature of six components. Therefore these equations were written in matrices form, which lead to a reduced representation of constitutive relations matrix. This matrix have ten non-zero independent elements, which represents the constants of piezoceramic materials. i.e. it contains five elastic constants, three piezoelectric constants, and two dielectric constants. These physical constants are tensor 
quantities, which given two subscript indices one of them is related to the direction of applied stimulus and the other to the perpendicular direction [3]

$$
\left[\begin{array}{l}
S_{1} \\
S_{2} \\
S_{3} \\
S_{4} \\
S_{5} \\
S_{6} \\
D_{1} \\
D_{2} \\
D_{3}
\end{array}\right]=\left[\begin{array}{cccccc|ccc}
S_{11}^{E} & S_{12}^{E} & S_{13}^{E} & 0 & 0 & 0 & 0 & 0 & d_{13} \\
S_{12}^{E} & S_{11}^{E} & S_{13}^{E} & 0 & 0 & 0 & 0 & 0 & d_{13} \\
S_{13}^{E} & S_{13}^{E} & S_{33}^{E} & 0 & 0 & 0 & 0 & 0 & d_{33} \\
0 & 0 & 0 & S_{55}^{E} & 0 & 0 & 0 & d_{15} & 0 \\
0 & 0 & 0 & 0 & S_{55}^{E} & 0 & d_{15} & 0 & 0 \\
0 & 0 & 0 & 0 & 0 & 2\left(S_{11}^{E}-S_{12}^{E}\right) & 0 & 0 & 0 \\
\hline 0 & 0 & 0 & 0 & d_{15} & 0 & \varepsilon_{11}^{T} & 0 & 0 \\
0 & 0 & 0 & d_{15} & 0 & 0 & 0 & \varepsilon_{11}^{T} & 0 \\
d_{13} & d_{13} & d_{33} & 0 & 0 & 0 & 0 & 0 & \varepsilon_{33}^{T}
\end{array}\right] \times\left[\begin{array}{c}
T_{1} \\
T_{2} \\
T_{3} \\
T_{4} \\
T_{5} \\
T_{6} \\
E_{1} \\
E_{2} \\
E_{3}
\end{array}\right]
$$

Table (1): Description of symbols used in constitutive relations.

\begin{tabular}{|c|c|c|c|}
\hline symbols & description & Type & Units \\
\hline $\mathrm{T}$ & Stress & Mechanical & $\mathrm{N} / \mathrm{m}^{2}$ \\
$\mathrm{~S}$ & Strain & Mechanical & $\mathrm{m} / \mathrm{m}$ \\
$\mathrm{E}$ & Electric field strength & Electrical & $\mathrm{V} / \mathrm{m}$ \\
$\mathrm{D}$ & Dielectric displacement & Electrical & $\mathrm{C} / \mathrm{m}^{2}$ \\
$\mathrm{~s}$ & Elastic compliance & Mechanical & $\mathrm{m}^{2} / \mathrm{N}$ \\
$\mathrm{c}$ & Elastic stiffness & Mechanical & $\mathrm{N} / \mathrm{m}^{2}$ \\
$\varepsilon$ & Permittivity & Electrical & $\mathrm{F} / \mathrm{m}$ \\
$\beta$ & Dielectric impermeability & Electrical & $\mathrm{m} / \mathrm{F}$ \\
$\mathrm{d}$ & Piezoelectric charge -strain constant & Electromechanical & $\mathrm{C} / \mathrm{N} \mathrm{or} \mathrm{m} / \mathrm{V}$ \\
e & Piezoelectric dielectric-stress constant & Electromechanical & $\mathrm{C} / \mathrm{m}^{2}$ or N/Vm \\
g & Piezoelectric voltage -strain constant & Electromechanical & $\mathrm{m}^{2} / \mathrm{C}$ or Vm$/ \mathrm{N}$ \\
$\mathrm{H}$ & Piezoelectric charge-stress constant & Electromechanical & $\mathrm{N} / \mathrm{C}$ or V/m \\
\hline
\end{tabular}

The only non-zero strain component, $\mathrm{S}$ and the electric field $\mathrm{E}$ component are substituted into the institutive relations, equation (2) to get the stress tensor component, T, and dielectric displacement, D. Both T and D are then substituted into the equation of motion (wave equation) in order to estimate the particle velocity $\mathrm{v} \approx \partial \mathrm{u} / \partial \mathrm{t}$ of the medium and then the specific acoustic impedance $\Gamma=\mathrm{p} / \mathrm{v}$ of the medium can be evaluated. For the reverse solution procedure the following constants, $s=1 / \mathrm{c}, \beta=1 / \varepsilon, \mathrm{h}=1 / \mathrm{d}$, can be used instead [4].

\section{Static Behavior of Piezoceramic Transducer (generation action)}

The elastic, piezoelectric, and dielectric constants of piezoceramic plates are determined by solving constitutive relations under many considerations such as the orientation of the applied static stimulus, shape, size of piezoceramic plates [5]. 
Application of static stimulus (stresses or electrical voltage) is commonly used to give an indication of the elastic, dielectric, and piezoelectric constants that mentioned before (equation 1).

This indication can be made utilizing the direct piezoelectric effect (application of static stresses) or the converse effect (application of static electric field), since the piezoceramic plate responds only to longitudinal static stress $\mathrm{T}_{3}$ in $\mathrm{z}$ direction (no shear stresses), the plate will compressed in $\mathrm{z}$ direction causing a change in dipole moment and then an electrical charge, of density $\mathrm{D}_{3}$, appears on the electrodes.

Direct effect which is applied under conditions of zero electric field yields $D_{i}=d_{i p} T_{p}$. On the other hand the converse effect under conditions of zero stresses inside the sample yields $S_{p}=d_{i p} E_{i}$ and then d can be calculated, where i,p $=1,2,3$.

\section{Dynamic Behavior of Piezoceramic Transducer (Ultrasonic probes)}

When piezoceramic plate exposed to an alternating electric field it periodically oscillates in accordance with the applied field. In this case its behavior can be described by the variation of the electrical impedance with frequency of oscillation. There are two approaches to obtain the impedance of such resonator as electrical circuit impedance each of which was derived from the piezoceramic constitutive relations.

An easier approach based on the measurement of resonance $f_{s}$ and antiresonance $f_{p}$ frequencies of unloaded, unclamped large plate, which resonates in the thickness mode. In such case piezoceramic resonator plate can be consider as a pure electrical reactance its impedance given as;

$$
Z_{\text {electrical }}=\frac{t}{j \omega A \varepsilon_{33}^{S}}\left[1-\frac{K_{t}^{2} \tan \left(\frac{\omega}{4 f_{p}}\right)}{\frac{\omega}{4 f_{p}}}\right]
$$

where $\mathrm{t}, \mathrm{A}, \mathrm{k}, \mathrm{K}_{\mathrm{t}}^{2}$ are thickness, radiated area, wavenumber, and thickness mode coupling coefficient of the resonator respectively. $\varepsilon_{33}$ is dielectric permittivity at constant strain (clamped), and $\omega$ is the angular frequency. 
For applications in which the resonator and loaded mediums are specified, a more general approach can be used to realize the electrical impedance. This approach deal with the driving point impedance of a two terminal equivalent circuit [6], [7], since under the loading conditions, the resonator cannot be consider as a purely reactance impedance but it posses also resistive part. The electrical impedance can be estimated as,

$$
Z_{\text {electrical }}=\frac{V_{\text {elctrical }}}{I_{\text {electrical }}}=\frac{1}{j \omega C_{E}}\left[1-\frac{K_{t}^{2}}{j k t} S^{*}\right]
$$

where $\mathrm{C}_{\mathrm{E}}$ is the clamped capacitance of piezoceramic plate and $\mathrm{S}^{*}$ is a complex function in normalized load impedance $\Gamma / \rho c$ and $\pi \mathrm{t} / \lambda$, where $\Gamma$ is the specific acoustical impedance, $\rho c$ is the acoustical impedance of the insonified medium, and $\lambda$ is the generated wavelength. Normally the function $S^{*}(\Gamma / \rho c, \pi t / \lambda)$ written in the complex form as;

$$
S^{*}=S_{1}+j S_{2} \text {, then } Z_{\text {electrical }}=R+j X
$$

where, the functions $S_{1}$ and $S_{2}$ are given in [8], then equation (4) can be split into two parts the real part $\mathrm{R}$, and the Imaginary part $\mathrm{X}$ which are given by;

$$
\begin{aligned}
& R=\frac{K_{t}^{2}}{\omega C_{E} k t} S_{1} \\
& X=\frac{K_{t}^{2}}{\omega C_{E} k t}\left[S_{2}-\frac{k t}{K_{t}^{2}}\right]
\end{aligned}
$$

Once the specific acoustical impedance $\Gamma=\mathrm{p} / \mathrm{v}$ of the insonified medium is known, both the resonance and antiresonance frequencies, for given acoustically loaded resonator (piezoceramic material), can be obtained.

Although equation (4) accurately represents the electrical impedance of any loaded resonator, utility of this equation may be not so easy. This is due to the inherently difficulties of the used techniques and instrumentation (laser vibrometer) to realize the specific acoustical impedance of insonified mediums. Therefore another theoretical prediction of such impedance may be abstracted from 1D thickness mode resonator model [8], since the Laplacian transform of the resonator electrical impedance Z(s) given in the form; 


$$
Z_{\text {electrical }}(s)=\frac{1}{s C_{E}}\left[1-\frac{K_{t}^{2}}{s T}\left(\frac{K_{F} T_{F}}{2}+\frac{K_{B} T_{B}}{2}\right)\right]
$$

where the functions $\mathrm{K}_{\mathrm{F}}$ and $\mathrm{K}_{\mathrm{B}}$ are defined as,

$$
\begin{aligned}
& K_{F}(s)=\frac{\left(1-e^{-s T}\right)\left(1-R_{B} e^{-s T}\right)}{\left(1-R_{F} R_{B} e^{-2 s T}\right)} \\
& K_{B}(s)=\frac{\left(1-e^{-s T}\right)\left(1-R_{F} e^{-s T}\right)}{\left(1-R_{F} R_{B} e^{-2 s T}\right)}
\end{aligned}
$$

where, $\mathrm{s}$ is the Laplacian complex variable, $\mathrm{T}$ is the one way propagation time, $T_{F}$ and $T_{B}$ are the transmission coefficients from the front and back faces of plate. $R_{F}$ and $R_{B}$ are the reflection coefficients from the front and back faces of plate.

\section{Equivalent Circuit of PZT Resonator:}

Theoretical analysis of the operation of piezoceramic materials, which is capable of simplifying the work of the probe designer, is extremely important. There are many possibilities to present and analysis equivalent circuit of piezoceramic materials. However, the sufficiently simple and practical equivalent circuit that expressed only in lumped electrical parameters (resistance, capacitance, inductance, voltage, and current) will be desirable. Butterworth-Van Dyke (BVD) circuit [9] was founded as the best simple lumped equivalent circuit, that is fitting a typical piezoceramic transducer, since it deals with piezoceramic vibrating system of an isolated single resonance mode (thickness mode). A conventional often used BVD equivalent circuit of a piezoceramic resonator is shown in Fig (1-a). This simplified form of BVD equivalent circuit has only four independent constant $\left(C_{0}, C_{1}, L_{1}\right.$, and $\mathrm{R}_{1}$ ) and therefore cannot accurately represents the electrical impedance over the entire frequency band of interest because the effect of the included higher vibration modes.

In practice the piezoceramic materials have various sources of losses. Mechanical or vibration losses are expressed by the tangent $\tan \delta_{\mathrm{m}}$ of each resonance, since that, the mechanical loss is dependent upon mechanical strain amplitude. Therefore, a resistance $\mathrm{R}_{1}$ (motional resistance) is inserted in the mechanical branch of BVD equivalent circuits. However another resistance $R_{o}$ of frequency dependent value is added in parallel to the terminal of BVD 
equivalent circuit in order to represent dielectric loss, which depends upon electric field amplitude. Additional resistance $\mathrm{R}_{\mathrm{L}}$ is inserted in the mechanical branch to represent the radiation resistance of the irradiated insonified medium (acoustical or mechanical load) as shown in Fig (1-b). The lumped component of this circuit can be calculated according to [6] as follow;

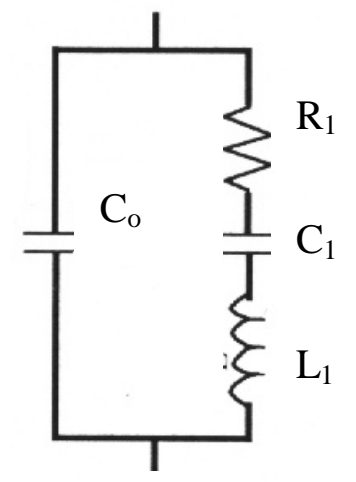

a

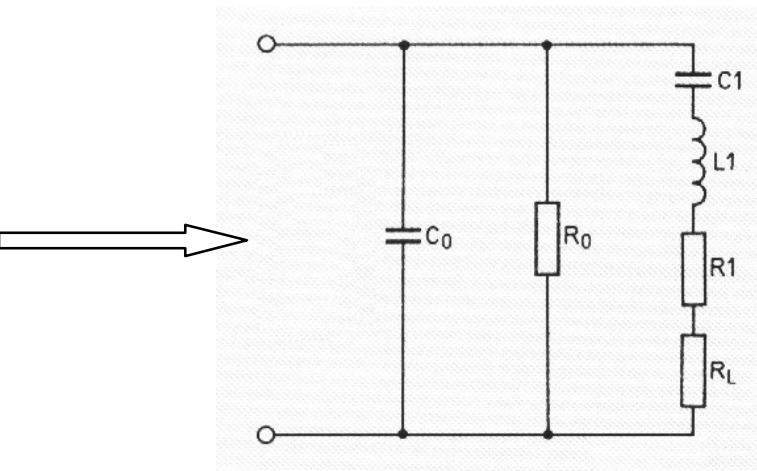

b

Fig. (1): BVD equivalent circuit, a) simple BVD equivalent circuit, b) modified BVD equivalent circuit with losses mechanisms

$$
\begin{aligned}
& R_{1}=\frac{1}{Q_{m} C_{o} \omega_{s}} \\
& Q_{m}=\frac{\left(L_{1} / C_{1}\right)^{\frac{1}{2}}}{R_{1}}
\end{aligned}
$$

where $R_{0}$ represents the dielectric loss, which is calculated from the loss tangent $\tan \delta_{\mathrm{E}}$ and series resonance frequency $\mathrm{f}_{\mathrm{s}}$, and $\mathrm{C}_{\mathrm{o}}$ is the capacitance of the clamped piezoceramic plate which is calculated from ;

$$
C_{o(\text { clamped })}=C_{\text {unclamped }}-C_{1(\text { motional })}
$$

where, $\mathrm{C}_{\text {unclamped }}$ may be obtained using equation (19).

The impedance of BVD circuit can be computed from first principle [10] to easily determine the series resonance frequency $f_{s}$ and parallel resonance frequency $f_{p}$ [11]-[12]. Fig. (2) illustrates the computed BVD equivalent circuit impedance and phase of F1 sample. It is clear that, the result maintains a good accuracy only in the vicinity of the resonant frequency. 


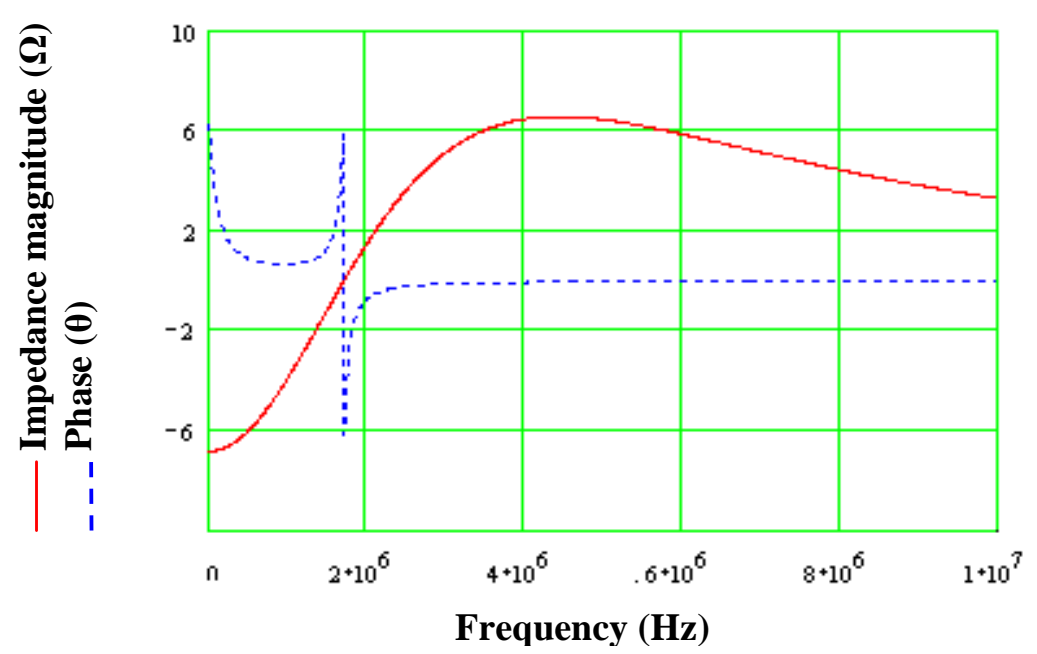

Fig. (2): Predicted electrical impedance of F1piezoceramic plate BVD model

The effect of various losses mechanisms, associated with piezoceramic materials, on the transduction efficiency taking into account by introducing the concept of complex piezoceramic material constants. In some certain applications of transduction devices the losses in piezoceramic medium are significant as compared with those in their components (matching layers, backing, etc...), since losses mechanisms greatly impact a device's efficiency, maximum drive-level and resonant characteristics. For instance the dielectric loss in the piezoceramic part in some probe, which operates under high pulse repetition frequency $(P R F)$, is responsible for the significant increase in the probe resistance at resonance frequency. Hence the electrical dissipation in such probes causing undesirable heat generation. Consequently great care must be taken during the design process.

According to the complex piezoceramic materials constants [13], the electrical component of the BVD equivalent circuit will be converted into complex form as;

$$
\begin{aligned}
& c_{33}^{S}=c_{33}^{S^{*}}-c_{33}^{S^{* *}} \\
& \varepsilon_{33}^{S}=\varepsilon_{33}^{S^{*}}+\varepsilon_{33}^{S^{* *}} \\
& C_{o}=C_{o}^{*}+C_{o}^{* *} \\
& C_{1}=C_{1}^{*}+C_{1}^{* *} \\
& L_{1}=L_{1}^{*}+L_{1}^{* *}
\end{aligned}
$$


where the superscripts $*$ and $* *$ respectively represent the real and imaginary part of the material constants.

\section{Predicting and Measuring the Resonator Parameters}

\subsection{Static Conditions}

For characterization of the used resonator plates F1-F7, Table (2), their static and dynamic actual parameters are investigated, since these parameters can be used in designation of ultrasonic probes.

Table (2): Sample Specifications.

\begin{tabular}{|c|ccc|}
\hline 5H plate name & Nominal frequency & Plate dimensions & Electrode \\
\hline F1 & $5 \mathrm{MHz}$ & $20 \times 20 \mathrm{~mm}$ & Silver \\
F2 & $5 \mathrm{MHz}$ & $20 \times 20 \mathrm{~mm}$ & Silver \\
F3 & $5 \mathrm{MHz}$ & $15 \times 15 \mathrm{~mm}$ & Silver \\
F4 & $5 \mathrm{MHz}$ & $15 \times 15 \mathrm{~mm}$ & Silver \\
F5 & $5 \mathrm{MHz}$ & $10 \times 10 \mathrm{~mm}$ & Silver \\
F6 & $5 \mathrm{MHz}$ & $10 \times 10 \mathrm{~mm}$ & Silver \\
F7 & $5 \mathrm{MHz}$ & $10 \times 10 \mathrm{~mm}$ & Silver \\
\hline
\end{tabular}

The generation action of piezoceramic PZT-5H rectangular plates, under the conditions of open circuit voltage (dielectric displacement $D$ is zero), was mathematically predicated using some typical tabulated values and apply for the relations (1), since under the open circuit voltage conditions $(D=0)$, the applied stress on the piezoceramic plate, in direction 3 , relates to the generated voltage signal given as,

$$
\frac{V}{t}=-g_{33} \times T_{3}
$$

$\mathrm{V} / \mathrm{t}$ is the electrical field strength $\left(\right.$ volt $/ \mathrm{m}^{2}$ ). From equation (13) piezoceramic plate F1 under the effect of 1 Newton compressive force (receiver) will generate voltage of $23 \mathrm{mV}$, while it will develops pressure of $-1.1 \mathrm{MPa}$ if F1 under the application of 10.6 volt (transmitter). Minus sign indicates that the generated pressure is compressive that accompanied with $-6.3 \mathrm{~nm}$ in thickness (transmitter).

Strain $\mathrm{S}_{33}$ developed into $\mathrm{F} 1$ in direction 3 due to application of 10.6 voltage in direction 3 (which generates stress of $-1.1 \times 10^{6} \mathrm{~N} / \mathrm{m}^{2}$ ) is given as $1.33 \times 10^{-5}$, hence, the elastic compliance $\mathrm{s}_{33}^{\mathrm{D}}$ can be calculated according to relation (1-c) where ; 


$$
\frac{\Delta t}{t}=-s_{33}^{D} \times T_{3}
$$

which give compliance constant of $1.1 \times 10^{-11} \mathrm{~m}^{2} / \mathrm{N}$. Knowing the volume Vol of the plate, it is easy to estimate the total stored energy $\zeta_{\mathrm{D}}$, which fed into the F1 PZT-5H plate by mechanical applied stress as follows;

$$
\zeta_{D}=\frac{V o l}{2} \times s_{33}^{D} \times T_{3}^{2}
$$

The calculated $\zeta_{\mathrm{D}}$ is as $1.4 \mu \mathrm{J}($ N.m). Table (3) collects the parameters of resonators behavior under static conditions.

Table (3): Action of Piezoceramic Plates as Generator.

\begin{tabular}{|c|cc|ccccc|}
\hline $\begin{array}{c}\text { Mechanical stimulus of } 1 \mathrm{~N} \\
\text { force (Receiver) }\end{array}$ & \multicolumn{4}{|c|}{ Electrical stimulus of 10.6 volt (Transmitter ) } \\
\hline Sample & voltage & Charge & $\begin{array}{c}\text { Stress } \\
\mathrm{T}_{33}\end{array}$ & $\Delta \mathrm{t}$ & $\begin{array}{c}\text { Strain } \\
\mathrm{S}_{33}\end{array}$ & compliance & $\begin{array}{c}\text { Energy } \\
\zeta_{\mathrm{D}}\end{array}$ \\
\hline $\mathrm{F} 1$ & $23 \mathrm{mV}$ & $0.25 \mu \mathrm{C}$ & $-1.1 \mathrm{MPa}$ & $-6.3 \mathrm{~nm}$ & $-1.3 \times 10^{-5}$ & $1.2 \times 10^{-11}$ & $1.4 \mu \mathrm{J}$ \\
$\mathrm{F} 2$ & $22 \mathrm{mV}$ & $0.23 \mu \mathrm{C}$ & $-1.2 \mathrm{MPa}$ & $-5.2 \mathrm{~nm}$ & $-1.1 \times 10^{-5}$ & $0.9 \times 10^{-11}$ & $1.2 \mu \mathrm{J}$ \\
$\mathrm{F} 3$ & $38 \mathrm{mV}$ & $0.17 \mu \mathrm{C}$ & $-1.2 \mathrm{MPa}$ & $-6.6 \mathrm{~nm}$ & $-1.5 \times 10^{-5}$ & $1.3 \times 10^{-11}$ & $0.9 \mu \mathrm{J}$ \\
$\mathrm{F} 4$ & $36 \mathrm{mV}$ & $0.17 \mu \mathrm{C}$ & $-1.3 \mathrm{MPa}$ & $-6.1 \mathrm{~nm}$ & $-1.5 \times 10^{-5}$ & $1.2 \times 10^{-11}$ & $0.9 \mu \mathrm{J}$ \\
$\mathrm{F} 5$ & $97 \mathrm{mV}$ & $0.05 \mu \mathrm{C}$ & $-1.1 \mathrm{MPa}$ & $-5.3 \mathrm{~nm}$ & $-1.1 \times 10^{-5}$ & $4.9 \times 10^{-11}$ & $1.5 \mu \mathrm{J}$ \\
$\mathrm{F} 6$ & $97 \mathrm{mV}$ & $0.06 \mu \mathrm{C}$ & $-1.1 \mathrm{MPa}$ & $-5.4 \mathrm{~nm}$ & $-1.1 \times 10^{-5}$ & $4.9 \times 10^{-11}$ & $1.5 \mu \mathrm{J}$ \\
$\mathrm{F} 7$ & $77 \mathrm{mV}$ & $0.08 \mu \mathrm{C}$ & $-1.4 \mathrm{MPa}$ & $-6.4 \mathrm{~nm}$ & $-1.6 \times 10^{-5}$ & $1.1 \times 10^{-11}$ & $0.4 \mu \mathrm{J}$ \\
\hline
\end{tabular}

Practically the charge constant $\mathrm{d}_{33}$ of seven PZT-5H piezoceramic plates was measured using a BERLINCOURT meter, which permits a direct measurement of $\mathrm{d}_{33}$ in the frequency range of $20-290 \mathrm{~Hz}$, picture (1). At these low frequencies (static), the electrical field $\mathrm{E}$ will consider constant and $\mathrm{d}_{33}$ is calculated as;

$$
d_{33}=\left(\frac{\partial D_{3}}{\partial T_{3}}\right)_{E}=\left(\frac{\partial S_{3}}{\partial E_{3}}\right)_{T}
$$

where $D_{3}$ and $E_{3}$ represent the charge density in $C / m^{2}$ and electric field in $V / m$ both in direction 3 respectively, while $S_{3}$ and $T_{3}$ represent the mechanical strain $\mathrm{m} / \mathrm{m}$ and stress $\mathrm{N} / \mathrm{m}^{2}$ both in direction 3 respectively. The subscript parameter $\mathrm{E}$ or $\mathrm{T}$ of brackets indicates that this calculation will be performed under constant $\mathrm{E}$ or $\mathrm{T}$. 


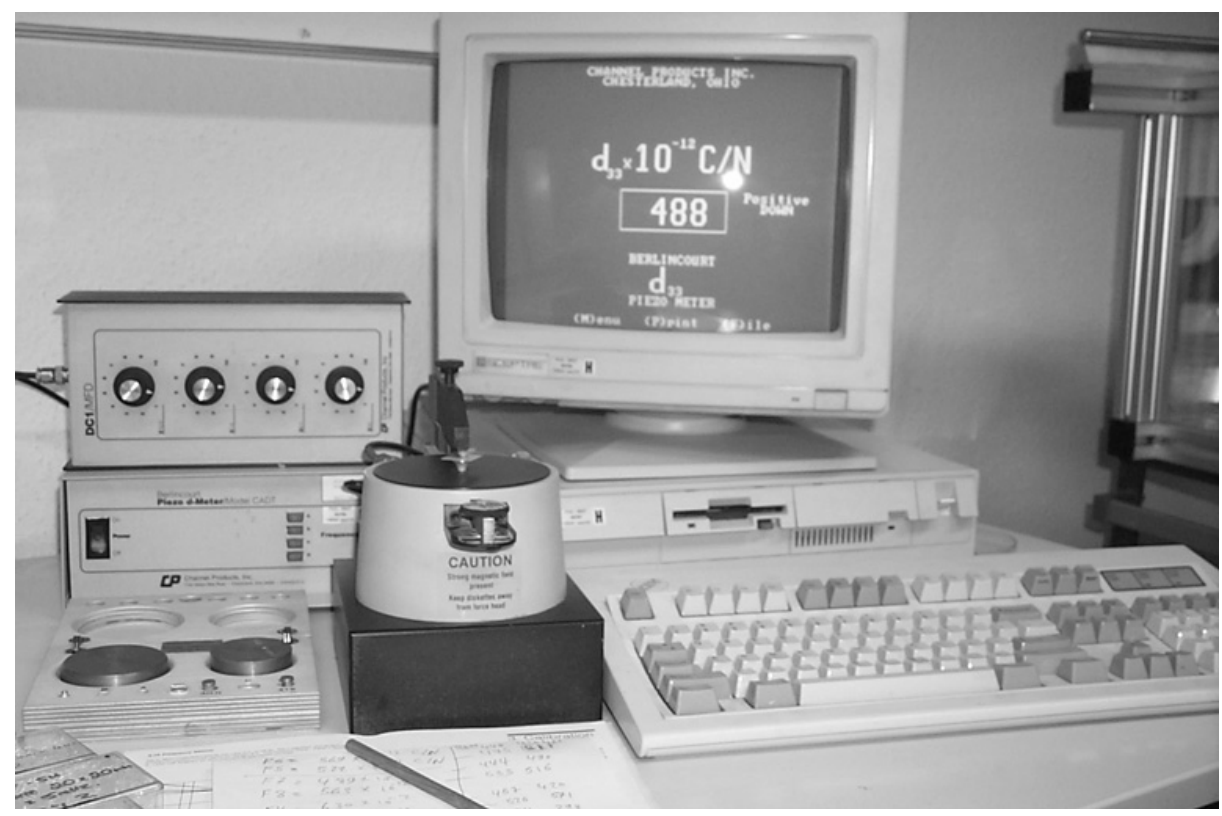

Picture (1): Berlincourt System for $\mathrm{d}_{33}$ measuring.

The average value of measured $d_{33}$ was tabulated in Table (4), since these average values may be used to estimate the other piezoceramic constants $\mathrm{g}_{31}$ and $\mathrm{g}_{15}$, where $\mathrm{g}_{\mathrm{xx}}$ represents the piezoceramic voltage constant, according to the equations

$$
\begin{aligned}
& g_{3 x}=\frac{d_{3 x}}{\varepsilon_{33}^{T}} \\
& g_{15}=\frac{d_{15}}{\varepsilon_{11}^{T}}
\end{aligned}
$$

Table (4): Measurements of charge constant $d_{33}$ at $30 \mathrm{~Hz}$ for different point on PZT samples.

\begin{tabular}{|c|ccccccc|}
\hline \multicolumn{7}{|c|}{$\mathrm{d}_{33} \times 10^{-12} \mathrm{C} / \mathrm{N}$} \\
\hline Sample/Point & Point 1 & Point 2 & Point 3 & Point 4 & Point 5 & mean & Typical \\
\hline F1 @ 30Hz & 411 & 584 & 566 & 533 & 535 & 525.8 & 593 \\
F2 @ 30Hz & 525 & 495 & 490 & 496 & 430 & 487.2 & 593 \\
F3 @ 30Hz & 633 & 611 & 634 & 633 & 600 & 622.2 & 593 \\
F4 @ 30Hz & 554 & 620 & 583 & 595 & 520 & 574.4 & 593 \\
F5 @ 30Hz & 480 & 460 & 550 & 502 & 490 & 496.4 & 593 \\
F6 @ 30Hz & 535 & 533 & 520 & 494 & 484 & 513.2 & 593 \\
\hline
\end{tabular}


Since the ferroelectric ceramics exhibit significant changes of $d_{33}$ with frequency, the BERLINCOURT system must be calibrated prior the measurement to obtain more accurate values of d's and g's., also values of $\mathrm{d}_{33}$ and $\varepsilon_{33}^{T}$ must be measured at the same frequency. This may be achieved by measuring the unclamped capacitance $\mathrm{C}$ of tested PZT-5H plate at the same frequency of measuring $\mathrm{d}_{33}$, and then applies to the following relation.

$$
\varepsilon_{33}^{T}=\frac{C t}{A}
$$

$t$ is the mean value of piezoceramic plate thickness in meter. The thickness $t$ was measured at different five locations on the surface of piezoceramic plate. Table (5) tabulates the measured values of thickness and area of piezoceramic plates.

Table (5): Measurements of thickness and area for different point on PZT samples

\begin{tabular}{|c|c|c|c|c|c|c|c|}
\hline \multirow[b]{2}{*}{ Sample/Point } & \multicolumn{7}{|c|}{ Average thickness of piezoceramic plates in $\mathrm{mm}$ and their area } \\
\hline & $\begin{array}{l}\text { Point } 1 \\
\text { mm }\end{array}$ & $\begin{array}{c}\text { Point } 2 \\
\mathrm{~mm}\end{array}$ & $\begin{array}{c}\text { Point } 3 \\
\mathrm{~mm}\end{array}$ & $\begin{array}{c}\text { Point } 4 \\
\text { mm }\end{array}$ & $\begin{array}{l}\text { Point } 5 \\
\text { mm }\end{array}$ & $\begin{array}{c}\text { mean } \\
\mathrm{mm}\end{array}$ & $\begin{array}{c}\text { area } \\
\mathrm{m}^{2}\end{array}$ \\
\hline & 0.4697 & 0.4697 & 0.4813 & 0.4792 & 0.4747 & 0.4749 & $4 \times 10-4$ \\
\hline $\mathrm{F} 2(20 \mathrm{x}$ & 0.4545 & 0.4488 & 0.4441 & 0.4553 & 0.4637 & 0.4533 & $4 \times 10-4$ \\
\hline $\mathrm{F} 3{ }_{(15 \times 15)}$ & 0.4525 & 0.4613 & 0.4486 & 0.4437 & 0.4568 & 0.4526 & $2.3 \times 10-4$ \\
\hline $\mathrm{F} 4{ }_{(15 \times 15)}$ & 0.4224 & 0.4220 & 0.4155 & 0.4148 & 0.4204 & 0.4190 & $2.3 \times 10-4$ \\
\hline & 0.4942 & 0.4914 & & & & 0.4935 & $1 \times 10-4$ \\
\hline$F 6_{(10 \times 10)}$ & 0.4934 & 0.4938 & 0.4992 & 0.4940 & 0.4954 & 0.4952 & $1 \times 10-4$ \\
\hline
\end{tabular}

Normally $\varepsilon_{33}^{T}$ is expressed in relative to the permittivity of free space which equal $8.85 \times 10^{-12} \mathrm{~F} / \mathrm{m}$. The calculated values of free $\varepsilon_{33}^{T}$ and relative $\varepsilon_{33}^{T}$ were tabulated in Table (6).

Table (6): Calculated of $\varepsilon_{33}^{T}$ at $30 \mathrm{~Hz}$ for different point on PZT samples.

\begin{tabular}{|c|ccccccc|}
\hline & \multicolumn{7}{|c|}{$\varepsilon_{33}^{T} \times 10-8 \mathrm{~F} / \mathrm{m}$} \\
\cline { 2 - 8 } Sample & $\mathrm{C}_{\mathrm{s}}$ & $\mathrm{C}_{\mathrm{p}}$ & $\mathrm{C}_{\text {unclampd }}$ & mean t & area & $\varepsilon_{33}^{T}$ & relative \\
& $\mathrm{nF}$ & $\mathrm{nF}$ & $\mathrm{F}$ & $\mathrm{m}$ & $\mathrm{m}^{2}$ & $\mathrm{~F} / \mathrm{m}$ & ----- \\
\hline $\mathrm{F} 1 @ 30 \mathrm{~Hz}$ & 22.04 & 22.03 & $2.2 \times 10-8$ & $4.7 \times 10-4$ & $4 \times 10-4$ & $2.6 \times 10-8$ & 2925.6 \\
$\mathrm{~F} 2 @ 30 \mathrm{~Hz}$ & 23.94 & 22.93 & $2.4 \times 10-8$ & $4.5 \times 10-4$ & $4 \times 10-4$ & $2.7 \times 10-8$ & 3042.6 \\
$\mathrm{~F} 3 @ 30 \mathrm{~Hz}$ & 13.89 & 13.98 & $1.4 \times 10-8$ & $4.5 \times 10-4$ & $2.3 \times 10-4$ & $2.7 \times 10-8$ & 3070.7 \\
$\mathrm{~F} 4 @ 30 \mathrm{~Hz}$ & 14.53 & 14.53 & $1.6 \times 10-8$ & $4.2 \times 10-4$ & $2.3 \times 10-4$ & $2.7 \times 10-8$ & 2998.1 \\
$\mathrm{~F} 5 @ 30 \mathrm{~Hz}$ & 5.35 & 5.34 & $5.4 \times 10-8$ & $4.9 \times 10-4$ & $1 \times 10-4$ & $2.6 \times 10-8$ & 2959.4 \\
$\mathrm{~F} 6 @ 30 \mathrm{~Hz}$ & 5.41 & 5.42 & $5.4 \times 10-8$ & $5 \times 10-4$ & $1 \times 10-4$ & $2.7 \times 10-8$ & 30.59 .3 \\
\hline
\end{tabular}




\section{2) Dynamic Conditions}

In the previous section the electrical and mechanical interaction of piezoceramic material in term of piezoelectric, dielectric, and elastic constants were derived through the constitutive relations. This implemented when the applied electrical signal (transmitter) or the applied acoustical pressure (receivers) were studied in static or quasi-static cases, namely the applied stresses (electrical or mechanical) have low frequencies $\leq 1 \mathrm{kHz}$. When these materials exposed to an alternative influences (electrical or mechanical) of high frequencies, which lies in the vicinity of its series mechanical resonance, its behavior can be characterized as a variation of electrical impedance with frequency. The main practical parameters, which normally measurable are $f_{s}, f_{p}$, and the quality factor $\mathrm{Q}_{\mathrm{m}}$, since piezoceramic plate behaves as inductance inbetween $\mathrm{f}_{\mathrm{s}}$ and $\mathrm{f}_{\mathrm{p}}$, Fig. (3), while it behaves as capacitance elsewhere.

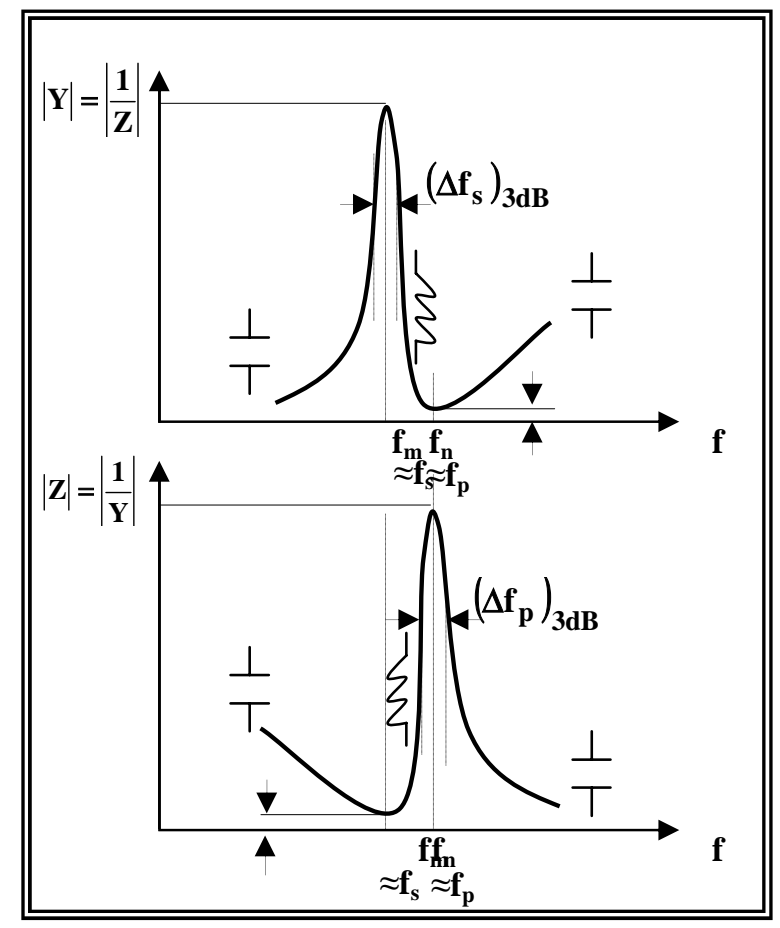

Fig.(3): Resonator electrical impedance as a dynamic behavior representation

Variation of such impedance can be traced by handling a 1D thickness mode model of each piezoceramic plate, namely equation (6) can be used to mathematically predict the electrical impedance of each resonator. Therefore, in order to put equation (6) in proper form, it is necessary to replace $Z_{\text {electrical }}$ from s-plan to w-plan. This can be done by diverted $Z(s)$ into frequency 
domain, $\mathrm{Z}(\mathrm{w})$, through the transformation of Laplacian complex variable s. the predicted impedance of $\mathrm{F} 1$ plate under front and back load conditions of 3.2 and 10 Mrayls respectively was illustrate Fig. (4).

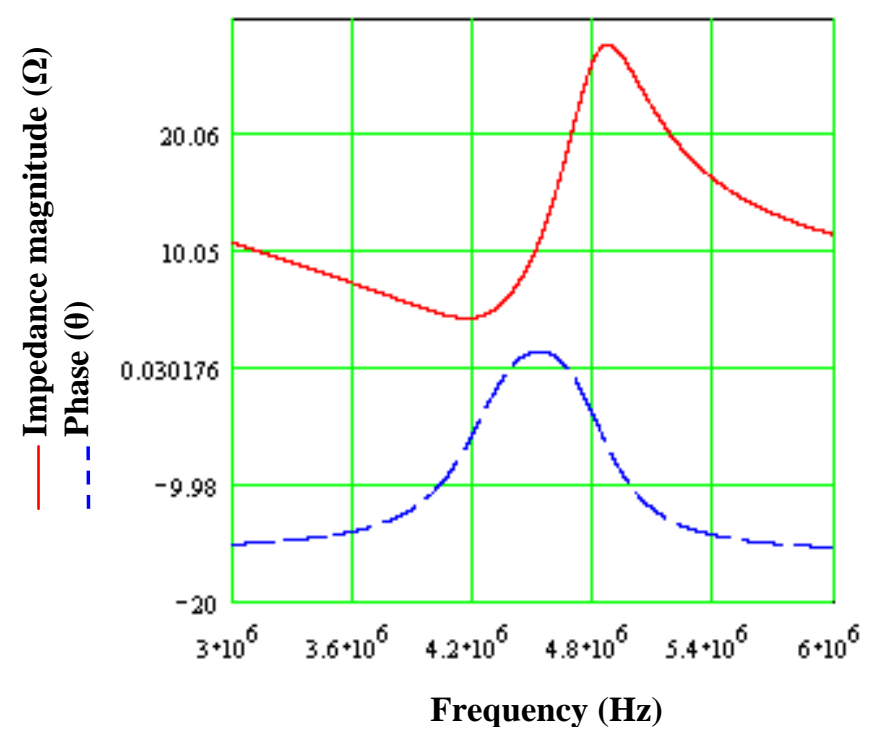

Fig. (4): Predicted electrical impedance of F1piezoceramic plate.

The complex functions $S_{1}$ and $S_{2}$ can be estimated by inspection of equation (4) and the diverted impedance $\mathrm{Z}(\mathrm{w}$ ) as follows;

$$
\begin{aligned}
& S_{1}(w)=\frac{K_{F}(w) T_{F}}{2} \\
& S_{2}(w)=\frac{K_{B}(w) T_{B}}{2} \\
& T=\frac{k t}{\omega}
\end{aligned}
$$

Variation of such impedance can be also measured using an impedance analyzer instrument, since a network analyzer $h p$ 4195A was used to measure the driving point electrical impedance of loaded F1 piezoceramic plates, Fig.(5) which can be compared with the predicted impedance, Fig.(4). Fig. $(6,7,8)$ illustrate the measured driving point electrical impedance of unloaded F1, F5, F7 piezoceramic plates respectively, where $f_{s}$ and $f_{p}$ were accurately determined. 


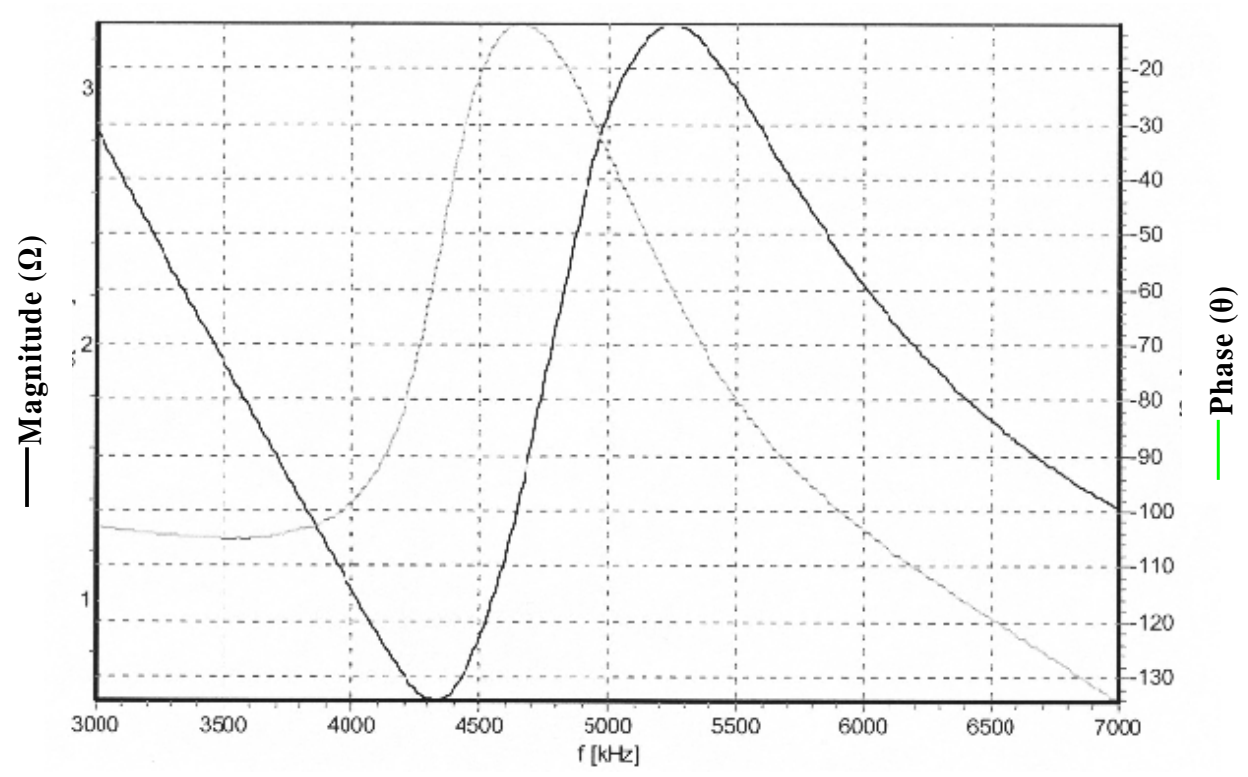

Fig. (5): Measured electrical impedance of F1piezoceramic plate under loaded condition.

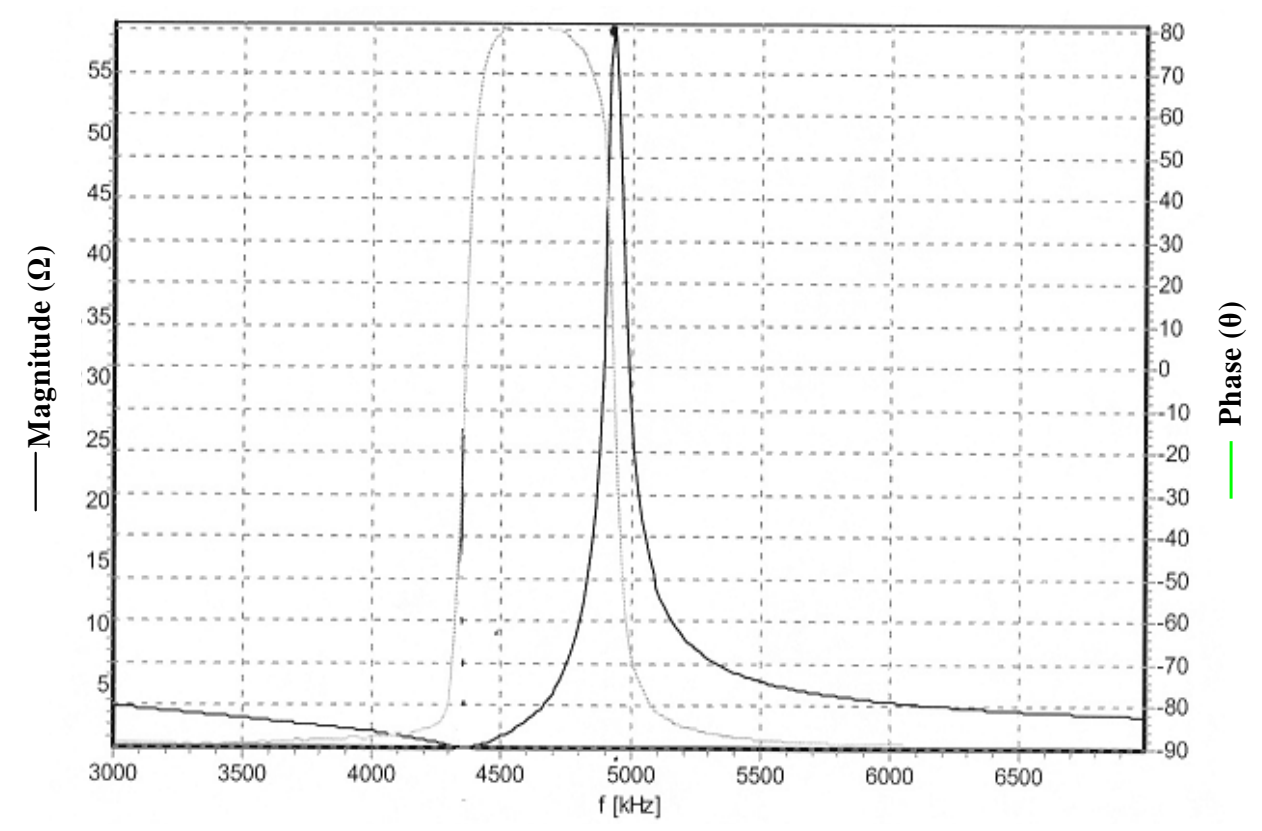

Fig. (6): Measured electrical impedance of F1piezoceramic plate unloaded condition. 


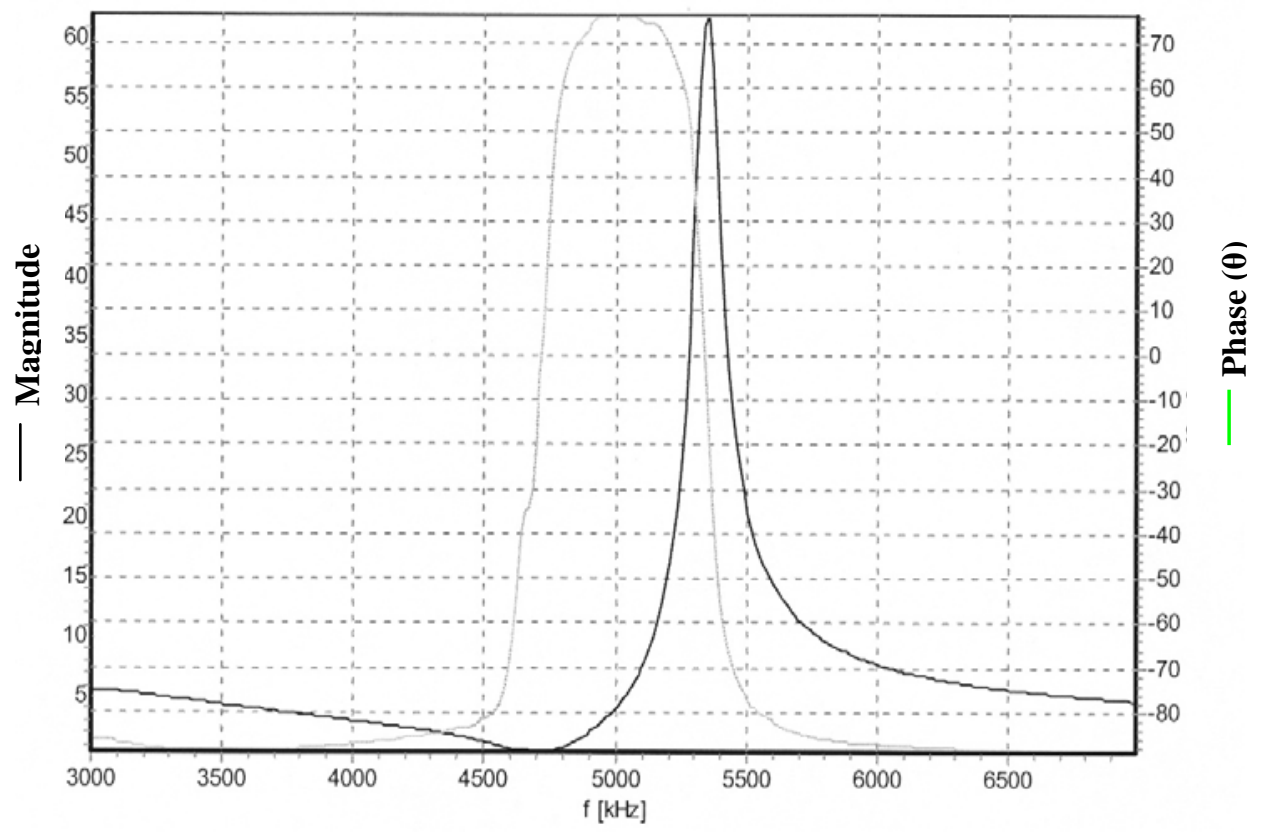

Fig. (7): Measured electrical impedance of F5 piezoceramic plate unloaded condition

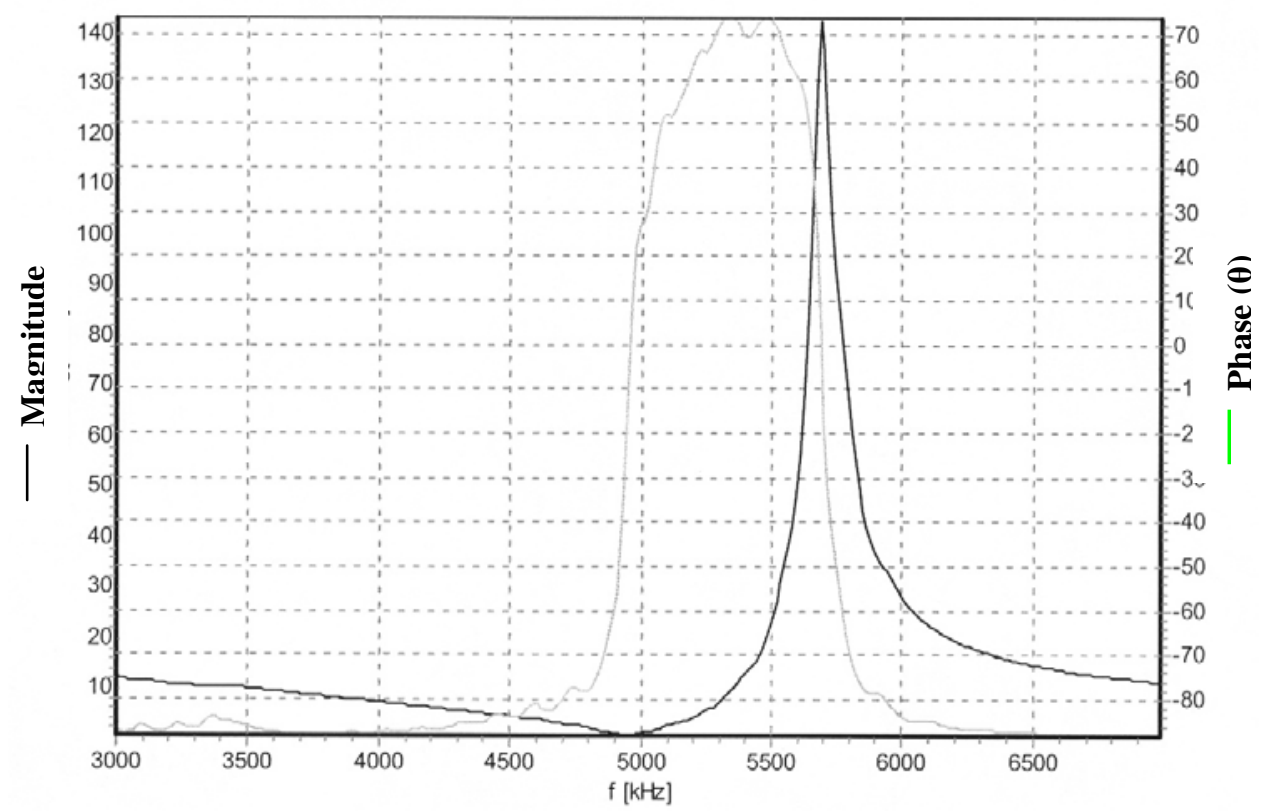

Fig. (8): Measured electrical impedance of F7 piezoceramic plate unloaded condition 


\section{Discussion:}

The most demand task of piezoceramic materials is their ability to converts all electrical energy into acoustic energy independent of frequency or vice versa. Practically, the transducer materials (piezoelectric ceramics) and their dimensions prevent these ideals from being satisfied, where for given piezoceramic materials only its volume and the applied stress govern the energy conversion (equation 15). Hence, in particular application when the applied force is limited, the energy generated can be increased by choosing a smaller surfaces area. Fig. (9) illustrates this concept of variation of stored energy and developed voltage with plate area.

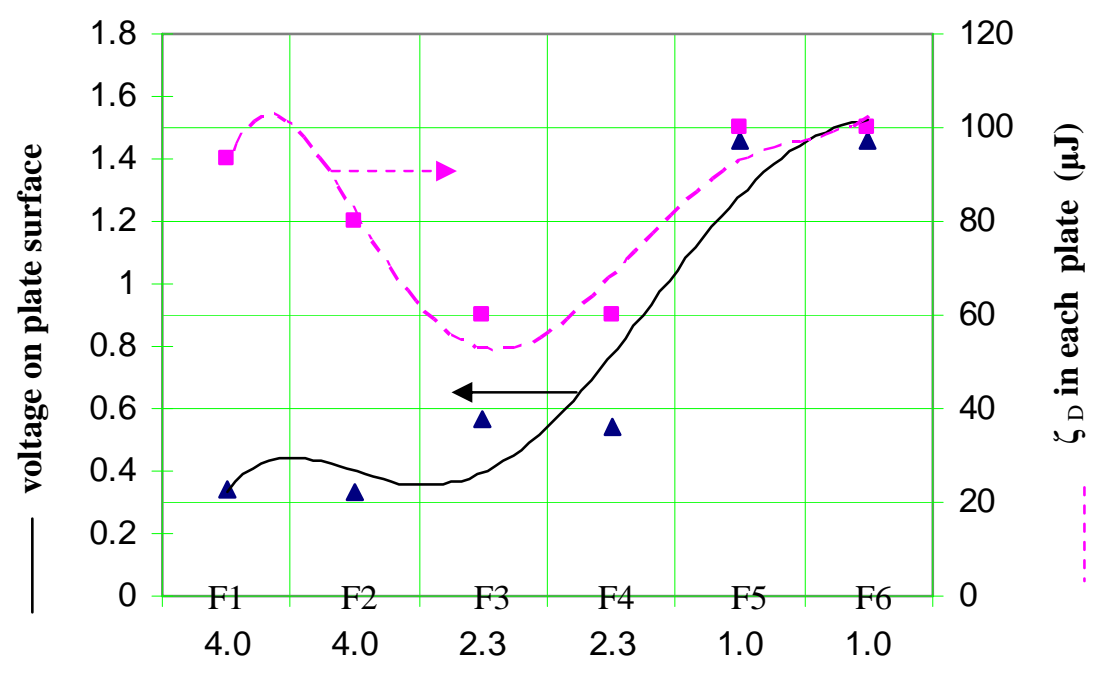

Area of different resonator plates $\times 10^{-4}\left(\mathrm{~m}^{2}\right)$

Fig. (9): Variation of developed voltage and stored energy with plate area

The approximately same degree of the energy absorbed within the piezoceramic materials being the advantage of PZT-5H mixture. This is due to its nearly equal values of dielectric loss $\tan \delta_{\mathrm{E}}$ and the mechanical loss $\tan \delta_{\mathrm{m}}$. Therefore, materials of slightly higher mechanical $\mathrm{Q}_{\mathrm{m}}$ and significantly higher sensitivity (higher $Q_{E}$ ) will be selected in order to improve the sensitivitybandwidth product.

Although, the behavior of piezoceramic materials may be considered strictly linear with very low electrical or mechanical excitation, excitation of high level signal produces high strain components in such materials causing a 
nonlinear behavior. Dependence of losses mechanisms on the excitation level has been interpreted as follows; high electrical drive (transmitter) increases linearly dielectric displacement $\mathrm{D}$ and then increases dielectric loss $\tan \delta_{\mathrm{E}}$, while high mechanical drive (receiver) will increases the mechanical loss tan $\delta_{\mathrm{m}}$.

Deviation of predicted impedance from the measured one under the same conditions demonstrates that utilizing of complex material constants greatly improves the ability to calculate the real part of impedance. Hence the variation in piezoceramic material response with frequency was found to produce better fit to the measured data by applying these complex constant to the equivalent circuit, which simulate performance of the thickness mode resonator.

Although piezoelectric materials are useful when operating in many varied modes, the calculated and measured piezoelectric material constants (table3) exhibit colinearity of the developed mechanical strain and the applied electric field intensity vectors, which is dominant in pure thickness mode vibration. However thickness vibration mode will be usually accompanied by either radial or planer modes, since piezoceramic exhibits both type of vibration modes at the same time, Fig. (10), i.e. a contraction expansion in the thickness direction is accompanied by expansion in the lateral direction and vice versa .Consequently the shape and size of piezoceramic plates, for ultrasonic probe designation, will be controlled in such away to reduce the other vibration modes, since the mode principally use for generation of the higher ultrasonic frequencies is the nearly pure thickness mode.

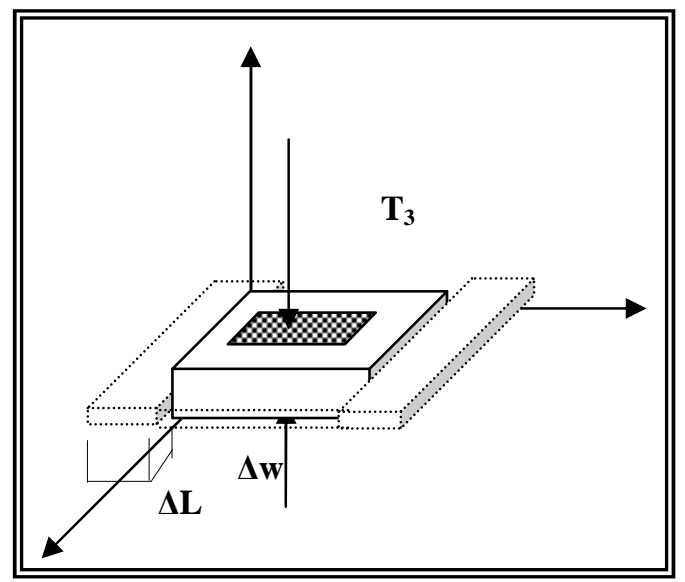

Fig. (10): Contraction in thickness direction accompanied by expansion in the lateral direction 


\section{Conclusion:}

Generally, as discussed before, the variability of piezoceramic parameters may be used to estimate the probabilities of the designed ultrasonic probes malfunctions. Therefore, evaluation and measurement of the electrical impedance and loss of piezoceramic transducer, considering the concept of complex piezoceramic materials constants, against frequency are significantly demand. Assessment procedure of the ultrasonic probe components, before the prototyping state, is greatly necessary.

\section{References:}

1. Dragan Damjanovic, P. Muralt and N. Setter, IEEE Sensors Journal, 1 (3), 1039, (2001).

2. M. J. Zipparo, K. K. Shung and T. R. Shrout, IEEE Trans. Ultrason. Ferroelect. ferq. contr., 44, 1038 (1997).

3. B.A. Auld, "Acoustic Fields and Waves in Solids_", vol. 1, John Wiley\& Sons (1973).

4. IEC 60483 International Standard, "Guide to dynamic measurements of piezoelectric ceramics with high electromechanical coupling" (1976).

5. American National Standards Institute "IEEE Standard on Piezoelectricity" 176 (1978).

6. W. Roth, Proceedings of the IRE, pp750-758, July (1949).

7. Kuo-Tsi Chang and Minsun Ouyang, Ultrasonics 41, 15 (2003).

8. G. Hayward, Ultrasonics (July), 153 (1984).

9. T. Ikada, "Fundamentals of Piezoelectricity", Chapter 7, p. 140, Oxford Univ. Press, (1990).

10. W. P. Mason, "Piezoelectric Crystals and their Application to Ultrasonic". New York: Van Nostrand, (1950).

11. J. W. Waanders, "Piezoelectric Ceramics", Phillips Applications Book, (1991).

12. G. E. Martin, J. Acoust. Soc. Amer. 26 (May), 413 (1954).

13. J.G. Smits, Ferroelectrics 64 (May), 275 (1985). 